\title{
Isotretinoin Treatment Practices and Outcomes in Acne Patients During the COVID-19 Pandemic: A Single Center, Retrospective, Comparative Study
}

\author{
Mehmet Fatih Atak ${ }^{1,2}$, Banu Ismail Mendi², Incilay Kalay Yildizhan², Hatice Sanli², \\ Banu Farabi ${ }^{3}$
}

1 Dermatology Department, Tokat State Hospital, Tokat, Turkey

2 Dermatology Department, Ankara University, Ankara, Turkey

3 Department of Internal Medicine, Saint Peter's University Hospital, New Brunswick, NJ, USA

Key words: Isotretinoin, treatment, acne, COVID-19, pandemic

Citation: Atak MF, Ismail Mendi B, Kalay Yildizhan I, Sanli H, Farabi B. Isotretinoin treatment practices and outcomes in acne patients during the covid-19 pandemic: a single center, retrospective, comparative study. Dermatol Pract Concept. 2022;12(1):e2022036. DOI: https://doi.org/10.5826/dpc.1201a36

Accepted: August 30, 2021; Published: January 2022

Copyright: (C2022 Atak et al. This is an open-access article distributed under the terms of the Creative Commons Attribution-NonCommercial License (BY-NC-4.0), https://creativecommons.org/licenses/by-nc/4.0/, which permits unrestricted noncommercial use, distribution, and reproduction in any medium, provided the original authors and source are credited.

Funding: None.

Competing interests: None.

Authorship: All authors have contributed significantly to this publication. MFA: Conceptualization, data curation, formal analysis, methodology, resources, validation, writing-original draft, writing review \& editing; BF: Methodology, resources, supervision, validation, writing-original draft, writing review \& editing; BI: Data curation, resources; IKY: Writing-review \& editing; HS: Revision of the content

Corresponding author: Banu Farabi, Department of Internal Medicine, Saint Peter's University Hospital, New Brunswick, NJ, USA. E-mail: banufarabi91@gmail.com

\footnotetext{
ABSTRACT Introduction: The COVID-19 pandemic drastically changed the priorities in healthcare services; outpatient management of acne has changed during this period.

Objectives: We aimed to investigate treatment practices, outcomes and identify modified follow-up schedules applied during the pandemic.

Methods: The patients who were admitted to dermatology outpatient clinic between March 13 and July 13, 2020, were included. Patients who were admitted between March 13 and July 13, 2019, were served as controls for the study. For each patient, age, gender, treatment protocols, treatment intervals, compliance with the treatment, treatment modifications, and adverse events were recorded.

Results: The total number of acne patients admitted to dermatology outpatient clinics during the pandemic period was 278 and consisted of $12.3 \%$ (278) of all admissions. Isotretinoin treatment was started in only $16(5.8 \%)$ of the patients. The proportion of patients who were under follow-up was significantly higher during the pandemic period $(\mathrm{P}<0.005)$. There was no difference between the pandemic period and the non-pandemic period in terms of starting isotretinoin treatment $(\mathrm{P}>0.05)$. During pandemic period, $79 \%$ of the patients who used isotretinoin were followed-up every two or more months. Extended follow-up intervals showed no difference for detecting side effects $(\mathrm{P}>0.05)$.
} 
Conclusions: Acne patients constitute an important part of dermatology outpatient clinics. During the pandemic period, majority of acne patients came for follow-up. Extended follow-up periods were adopted by physicians and were found safe and effective in the current study. Thus, isotretinoin treatment seems efficacious and safe during pandemic period.

\section{Introduction}

COVID-19 pandemic affected the healthcare practices all over the world in 2020. It drastically changed the priorities in healthcare services, thus outpatient management of elective diseases such as acne have also been shaped during this period [1]. Restrictions and the fear of infection altered outpatient clinic patient profile in dermatology clinics and resulted implementation of teledermatology visits [2].

The effects of pandemic on dermatological practices have been investigated especially in chronic diseases treating with immunosuppressive or immunomodulatory drugs, but there are not enough studies focusing on the effects of acne and its management during the pandemic. Acne patients consist of an important part of the routine dermatology outpatient clinics, thus further studies are required to establish the management of acne [3].

\section{Objectives}

We aimed to investigate the treatment practices, treatment outcomes, adherence of acne patients and to identify modified follow-up schedules during the pandemic period.

\section{Methods}

The IRB approval for the study was obtained from the Ankara University Ethics Committee on April 2021. The patients visited the dermatology clinic between March 13 and July 13, 2020, when first restrictions were imposed by the Turkish government due to pandemic, have been retrospectively reviewed for the clinical diagnosis of acne vulgaris through electronic medical record system. A total number of 278 acne patients seen in our clinic for 4 months period during the pandemic was included in the study. For each patient age, gender, treatment protocols, treatment intervals, compliance with the treatment, adverse events were recorded. Patients were stratified according to age as follow: younger than 18 years of age, and 18 years or older. Treatment protocols were assessed in three categories as follows: topical treatment, systemic antibiotic treatment with topical treatments, and oral isotretinoin treatment. Treatment intervals were determined in two different categories as follows: monthly or in every two months or longer. Adverse events were recorded. The patients admitted in the outpatient clinic between March 13 and July 13, 2019, served as controls for the study.

Statistical analysis was performed using the SPSS 22.0 program. Mean, standard deviation and percentage were used for descriptive statistics. Chi-square test was performed to examine differences between categorical variables in same groups and the $\mathrm{P}$ value of less than 0.05 was accepted as statistically significant.

\section{Results}

The total number of patients admitted to outpatient clinic during pandemic period was 2265. Acne patients consisted of $12.3 \%$ (278) of all admissions. On the other hand, the total number of patients admitted to dermatology outpatient clinic during non-pandemic period was 7604 and $7.5 \%$ (577) were acne patients. The distribution of the patients according to the gender and age is shown in Table 1 , and there was no significant difference between two periods according to gender and age $(\mathrm{P}>0.05)$.

Table 1. Demographic Characteristics of Patients Before and After The Pandemic Period

\begin{tabular}{|l|c|c|c|}
$\begin{array}{c}\text { Demographic characteristics of } \\
\text { the acne patients }\end{array}$ & Pandemic period (n = 278) & Non-pandemic period (n = 577) & P values \\
\hline Sex & $179(64.4 \%)$ & $383(66.4 \%)$ & 0.57 \\
\hline Male & $99(35.6 \%)$ & $194(33.6 \%)$ & 0.22 \\
\hline Female & $22.80(\mathrm{SD} \pm 7.12)$ & $22.16(\mathrm{SD} \pm 6.98)$ & 0.53 \\
\hline Age, years (mean) & $64(23 \%)$ & $122(21.1 \%)$ & \multirow{2}{*}{0} \\
\hline$<18$ & $214(87 \%)$ & $455(88.9 \%)$ & \\
\hline$>18$ & & & \\
\hline
\end{tabular}

SD: standard deviation. 
Table 2 summarizes the treatment modalities of the patients with the first admission and under follow-up in both pandemic and non-pandemic periods. Among the patients who applied to dermatology outpatient clinic during pandemic period, $124(44.6 \%)$ had first admission and 150 $(54 \%)$ were under follow-up for different acne treatments, and 4 of them were consultation patients. One hundred and sixteen $(93.5 \%)$ patients with first admission did not receive treatment for acne previously, whereas 8 of them were under systemic treatments (oral isotretinoin $\mathrm{n}=2$, doxycycline $n=6$ ). Of the 116 treatment naïve patients, topical treatment was given to $77(\mathrm{n}=27.7 \%)$, oral isotretinoin treatment to $16(5.8 \%)$ and systemic antibiotic treatment with topicals to $23(8.3 \%)$ patients. Of the 8 patients taking systemic treatment at first admission, doxycycline treatment was switched to oral isotretinoin for 3 patients and to azithromycin for one patient, and the others continued their current systemic treatment (oral isotretinoin $\mathrm{n}=$, doxycycline $\mathrm{n}=2$ ).

During the pandemic, $26(9.4 \%)$ of the follow-up patients were under only topical treatments, 10 patients $(3.6 \%)$ were under systemic antibiotic treatment with topical treatment, and 114 patients $(41 \%)$ were under oral isotretinoin treatment. During this period, for 3 patients topical treatments were switched to oral isotretinoin because of the severity of acne and 1 of the patients under doxycycline treatment was switched to isotretinoin due to lack of efficacy. The rate of total acne patients treated with isotretinoin during the pandemic period was 50\% (139 patients). Of these patients, 114 $(41 \%)$ were follow-up ones, $16(5.8 \%)$ of them were started isotretinoin treatment in their first visits.

The number of patients with first admission was statistically higher in non-pandemic period and the number of follow-up patients was statistically higher in pandemic period
$(\mathrm{P}<0.05)$. There was no statistically significant difference in the two periods according to the number of treatment naïve patients, the first admission patients previously treated with any systemic agent, and patients to whom was given systemic treatment in first admission $(\mathrm{P}>0.05)$. The rate of acne patients treated with isotretinoin during the pandemic period and non-pandemic period were $50 \%$ and $\% 39.3$, respectively. Isotretinoin use was found significantly higher in the pandemic period $(P=0.003)$. There was no statistically significant difference between the pandemic period and the non-pandemic period in terms of starting isotretinoin treatment in patients with first admission $(\mathrm{P}>0.05)$. There was no significant difference for using isotretinoin treatment in patients who were under follow-up in the two groups $(\mathrm{P}=0.17)$.

Follow-up intervals, drop-out rates and reported adverse events in patients treated with isotretinoin during pandemic and non-pandemic period are given in Table 3. The rate of patients who used isotretinoin and dropped out from follow-up during the pandemic period was significantly higher than the control group ( $\mathrm{P}<0.001)$. During the pandemic period, the rate of patients using isotretinoin, which was followed in every two or more-months, was significantly higher than the control group $(\mathrm{P}<0.001)$. Side effects in pandemic and non-pandemic period were seen in $10.1 \%$ and $19.8 \%$ of the patients, respectively. The rate of patients using isotretinoin who developed side effects was found significantly higher in the non-pandemic period compared to the pandemic period $(\mathrm{P}=0.01)$.

In the 4-month pandemic period (March-July 2020), only two patients were diagnosed with SARS-Cov-2 infection. One of the patients (aged 27 years) was using isotretinoin and she only showed mild upper respiratory tract infection symptoms. Other patient (aged 39 years) was using topical treatments. Neither of them was hospitalized.

Table 2. Treatment Status of Patients Admitted During the Pandemic and Non-Pandemic Period

\begin{tabular}{|l|c|c|c|}
\hline & Pandemic period (n= 278) & Non-Pandemic period (n= 577) & P values \\
\hline Patients with first admission & $124(44.6 \%)$ & $336(58.2 \%)$ & $<0.005$ \\
\hline Treatment naïve & $116(93.5 \%)$ & $318(94.6 \%)$ & 0.652 \\
\hline $\begin{array}{l}\text { Treated with any systemic agent } \\
\text { (antibiotics and oral isotretinoin) }\end{array}$ & $8(6.5 \%)$ & $18(5.4 \%)$ & 0.34 \\
\hline $\begin{array}{l}\text { Treatment naive patients started } \\
\text { systemic isotretinoin }\end{array}$ & $39(31.4 \%)$ & $129(38.3 \%)$ & $<0.005$ \\
\hline Follow-up patients & $150(54 \%)$ & $239(41.4 \%)$ & 0.17 \\
\hline Isotretinoin treatment & $114(76 \%)$ & $173(72.4 \%)$ & 0.06 \\
\hline $\begin{array}{l}\text { Systemic antibiotic treatment and } \\
\text { topical treatments }\end{array}$ & $10(6.7 \%)$ & $58(24.3 \%)$ & 0.06 \\
\hline Only topical treatments & $26(17.3 \%)$ & & $227(39.3 \%)$ \\
\hline Treatment status in all patients & $139(50 \%)$ & $103(17.9 \%)$ & 0.003 \\
\hline Isotretinoin treatment & $36(12.9 \%)$ & $247(42.8 \%)$ & 0.43 \\
\hline Systemic antibiotic and topicals & $103(37.1 \%)$ & 0.43 \\
\hline Only topical treatment & & & \\
\hline
\end{tabular}


Table 3. Changes in Follow-Up Intervals, Patient Drop-Out Rates, and Reported Adverse Events in Isotretinoin Patients

\begin{tabular}{|l|c|c|c|}
\hline \multicolumn{1}{|c|}{ Number of isotretinoin patients } & Pandemic period (n= 139) & Non-pandemic period (n = 227) & P values \\
\hline Followed-up for extended intervals & $79(56.8 \%)$ & $65(28.6 \%)$ & $<0.001$ \\
\hline Lost to follow-up & $30(21.6 \%)$ & $6(2.6 \%)$ & $<0.001$ \\
\hline Reported adverse events & $14(10.1 \%)$ & $45(19.8 \%)$ & 0.01 \\
\hline liver enzyme abnormality & $5(3.6 \%)$ & $10(4.4 \%)$ & $6(2.6 \%)$ \\
\hline hypertriglyceridemia & $2(1.4 \%)$ & $9(4 \%)$ & $3(1.3 \%)$ \\
\hline back pain & $2(1.4 \%)$ & $3(1.3 \%)$ & \\
\hline nasal bleeding & $2(1.4 \%)$ & $1(0.4 \%)$ & \\
\hline psychiatric symptoms & $1(0.8 \%)$ & $4(1.8 \%)$ & $7(3.1 \%)$ \\
\hline headache & $1(0.8 \%)$ & $1(0.4 \%)$ & \\
\hline severe xerosis-eczematous dermatitis & $1(0.8 \%)$ & $1(0.4 \%)$ & \\
\hline dry eye syndrome & - & - & \\
\hline photosensitivity & - & & \\
\hline anal fissure & & & \\
\hline
\end{tabular}

\section{Conclusions}

Novel coronavirus pandemic has caused significant changes in dermatology practices [3]. As depicted in our study, the accessibility to outpatient clinics and patient compliance with systemic treatments were affected by the pandemic. According to recent literature, acne patients consisted of the majority of the patients who are admitted to dermatology outpatient clinics [1], and treatment compliance was the highest in this group [3]. This might be due to that acne is relatively common condition in younger individuals and younger patients seek more care because of the psychosocial effect of the disease [4]. Additionally, the knowledge of the mild disease course of COVID-19 infection might cause young patients to reach hospital clinics without fear compared to elderly population ]. Another reason for increased percentage of acne patients can be intense use of masks, thus causing increased prevalence of 'mask acne', likely result of increased usage of personal protective equipment with the current pandemic ]. The percentage of acne patients admitted to dermatology outpatient clinic during the pandemic period was found significantly increased compared to non-pandemic period in the current study.

Starting from March 2020, social distancing measures and restrictions were imposed to prevent the spread of the virus by Turkish Authorities, however, hospital admissions were exempted. These restrictions were thought to not affect the admissions of acne patients. While the rate of acne patients admitted for their first visit was significantly low in pandemic period, the rate of patients who were admitted for follow-up visits was significantly higher. This is probably as a result of avoidance of hospital visits due to non-emergent conditions during the pandemic period and high treatment compliance rate of our patient population.
Theoretically, isotretinoin treatment may cause disruption of the basement membrane of the mucosal surfaces by causing mucosal dryness and thinning. This, hypothetically, can increase the risk of COVID-19 transmission [9]. There is no established guideline regarding the use of isotretinoin during the pandemic period. In the beginning of the pandemic British Association of Dermatologists recommended starting or continuing oral isotretinoin treatment where the risks are outweighed by the benefits with monthly follow-up [10]. According to this statement, if there is no risk of pregnancy, the prescriptions for several months could be given taking into account the need to monitor blood test and side effects with remote consultations. In situations where it is not possible to perform monthly pregnancy tests, home pregnancy tests are recommended as a suitable alternative [11]. With the rapid growth of teledermatology, these recommendations have been practiced and the patients were able get their prescriptions instead of in-person visits [2]. A recent study by Ruggiero et al reported high degree of satisfaction and well-being after teledermatology visits [12].

Between the pandemic and non-pandemic periods, there was no significant difference in the rate of starting systemic treatment for acne patients who were admitted for their first visit. Additionally, the percentage of patients using isotretinoin during pandemic period was significantly higher compared to non-pandemic period. This was due to a relative increase of the number of patients who were followed-up with their current isotretinoin treatment. In our clinic, isotretinoin therapy was not avoided during the pandemic period.

Isotretinoin treatment needs to be monitored in terms of both teratogenesis and bone marrow and metabolic side effects (leukopenia, hypertriglyceridemia, liver enzyme abnormalities) $[14,15])$. Although there are many studies that 
do not support monthly laboratory testing for isotretinoin [15], there are many centers that apply monthly laboratory follow-up in clinical practice, especially in terms of teratogenicity [16]. Monthly laboratory monitoring is also a common practice among physicians in our clinic. When we compared the monthly follow-up to in every two or more-month follow-up rate between the 2 groups, in every 2 or more-month follow-up the rate was higher during the pandemic period. Our retrospective analysis showed that extended follow-up intervals showed no significant difference for detecting side effects of isotretinoin. Additionally, the reported side effect rate was significantly lower during the pandemic period. This might be due to the lock-down and increased use of skin care products due to easy accessibility to emollients and other protective measures.

In the 4-month follow-up, only one patient under isotretinoin treatment had a positive SARS-Cov-2 PCR result and she showed only mild upper respiratory tract infection symptoms. Thus, isotretinoin treatment seems efficacious and safe during pandemic. We recommend extended intermittent laboratory follow-up to reduce the risk of SARS-Cov-2 transmission, however further large cohort studies are required to elaborate the risks.

Acne patients constitutes an important part of dermatology outpatient clinics. The proportion of acne patients was observed as significantly higher during the pandemic period. This most likely reflects young individuals seek for more care due to psychosocial effects of the disease and less fear of infection by SARS-Cov-2. Another reason for increased percentage of acne patients is intense use of masks, causing increased prevalence of 'mask acne', likely result of increased usage of personal protective equipment with the current pandemic. During pandemic period, the majority of acne patients was admitted for follow-up and physicians did not avoid using isotretinoin treatment in the follow-up patients. Extended follow-up periods were adopted by physicians and were found safe and effective in the current study. Thus, isotretinoin treatment seems efficacious and safe during pandemic.

\section{References}

1. Kutlu Ö, Güneş R, Coerdt K, Metin A, Khachemoune A. The effect of the "stay-at-home" policy on requests for dermatology outpatient clinic visits after the COVID-19 outbreak. Dermatol Ther. 2020;33(4):e13581. DOI: 10.1111/dth.13581. PMID: 32401401. PMCID: PMC7272831.

2. Sharma A, Jindal V, Singla P, Goldust M, Mhatre M. Will teledermatology be the silver lining during and after COVID-19? Dermatol Ther. 2020;33(4):e13643. DOI: 10.1111/dth.13643. PMID: 32441373. PMCID: PMC7267127.

3. Alshiyab DM, Al-Qarqaz FA, Muhaidat JM. Impact of COVID-19 pandemic on the continuity of care for dermatologic patients on systemic therapy during the period of strict lockdown. Ann Med Surg (Lond). 2020;60:571-4. DOI: 10.1016/j.amsu.2020.11.056. PMID: 33251007. PMCID: PMC7686756.

4. Aslan Kayiran M, Karadag AS, Jafferany M. Psychodermatology of acne: Dermatologist's guide to inner side of acne and management approach. Dermatol Ther. 2020;33(6):e14150. DOI: 10.1111/dth.14150. PMID: 32770727.

5. Pasion R, Paiva TO, Fernandes C, Barbosa F. The AGE Effect on Protective Behaviors During the COVID-19 Outbreak: Sociodemographic, Perceptions and Psychological Accounts. Front Psychol. 2020; 11:561785. DOI: 10.3389/fpsyg.2020.561785. PMID: 33178069 . PMCID: PMC7595956.

6. Searle T, Ali FR, Al-Niaimi F. Identifying and addressing "Maskne" in clinical practice. Dermatol Ther. 2021;34(1):e14589. DOI: 10.1111/dth.14589. PMID: 33244836.

7. Han C, Shi J, Chen Y, Zhang Z. Increased flare of acne caused by long-time mask wearing during COVID-19 pandemic among general population. Dermatol Ther. 2020;33(4):e13704. DOI: 10.1111/dth.13704. PMID: 32472634. PMCID: PMC7300566.8.

8. Damiani G, Gironi LC, Grada A, et al. COVID-19 related masks increase severity of both acne (Maskne) and rosacea (Mask rosacea): Multi-center, real-life, telemedical, observational prospective study. Dermatol Ther. 2021:e14848. DOI: 10.1111/dth.14848. PMID: 33533563. PMCID: PMC7995182.

9. Abdelmaksoud A, Vestita M, El-Amawy HS, et al. Isotretinoin therapy in the era of COVID-19. Dermatol Ther. 2020;33(4):e13482. DOI: 10.1111/dth.13482. PMID: 32358858. PMCID: PMC7261989.

10. British Association of Dermatologists. Guidance for Managing Patients on Isotretinoin During The Coronavirus Pandemic 2020. Available from: https://www.bad.org.uk/shared/get-file. ashx? itemtype $=$ document $\&$ id $=6661$.

11. Pathoulas JT, Farah RS, Fiessinger L, Mansh M. An opportunity for improvement: iPLEDGE policy changes during the coronavirus pandemic. Dermatol Ther. 2020;33(6):e14411. DOI: 10.1111/ dth.14411. PMID: 33052629. PMCID: PMC7646038.

12. Ruggiero A, Megna M, Annunziata MC, et al. Teledermatology for acne during COVID-19: high patients' satisfaction in spite of the emergency. J Eur Acad Dermatol Venereol. 2020;34(11):e662-e663. DOI: 10.1111/jdv.16746. PMID: 32534472. PMCID: PMC7323158.

13. Kim JK, Crimmins EM. How does age affect personal and social reactions to COVID-19: Results from the national Understanding America Study. PLoS One. 2020;15(11):e0241950. DOI: 10.1371/journal.pone.0241950. PMID: 33170903. PMCID: PMC7654776.

14. Dessinioti C, Zouboulis CC, Bettoli V, Rigopoulos D. Comparison of guidelines and consensus articles on the management of patients with acne with oral isotretinoin. J Eur Acad Dermatol Venereol. 2020;34(10):2229-2240. DOI: 10.1111/jdv.16430. PMID: 32277497.

15. Lee YH, Scharnitz TP, Muscat J, Chen A, Gupta-Elera G, Kirby JS. Laboratory Monitoring During Isotretinoin Therapy for Acne: A Systematic Review and Meta-analysis. JAMA Dermatol. 2016;152(1):35-44. DOI: 10.1001/jamadermatol.2015.3091. EPMID: 26630323.

16. Choi JS, Koren G, Nulman I. Pregnancy and isotretinoin therapy. CMAJ. 2013;185(5):411-413. DOI: 10.1503/cmaj.120729. PMID: 23296582. PMCID: PMC3602257. 